\title{
Changes of the first Schumann resonance frequency during relativistic solar proton precipitation in the 6 November 1997 event
}

\author{
V. C. Roldugin, Ye. P. Maltsev, A. N. Vasiljev, E. V. Vashenyuk \\ Polar Geophysical Institute, Academy, Apatity, Murmansk reg., 184200 Russia
}

Received: 23 September 1998 / Revised: 26 March 1999 / Accepted: 26 April 1999

\begin{abstract}
The variations of the first mode of Schumann resonance are analyzed using data from Kola peninsula stations during the solar proton event of 6 November 1997. On this day the intensive flux of energetic protons on GOES- 8 and the $10 \%$ increase of the count rate of the neutron monitor in Apatity between 1220 and 2000 UT were preceded by a solar X-ray burst at 1155 UT. This burst was accompanied by a simultaneous increase of the Schumann frequency by $3.5 \%$, and the relativistic proton flux increase was accompanied by $1 \%$ frequency decrease. These effects are explained by changes of the height and dielectric permeability of the Earth-ionosphere cavity.
\end{abstract}

Key words. Ionosphere (ionospheric disturbances; solar radiation and cosmic ray effects) $\cdot$ Radio science (ionospheric propagation)

\section{Introduction}

The cavity between the Earth and ionosphere is a resonator for electromagnetic waves. The Schumann resonance frequencies cover several $\mathrm{Hz}$. The simplest vacuum model confined with two concentric perfectly conducting spheres yields the frequency (Schumann, 1952)

$\omega_{n}=\frac{c}{a} \sqrt{n(n+1)\left(1-\frac{h}{a}\right)}$,

where $n$ is an integer number, $c$ is the light velocity, $a$ is the radius of the inner sphere, $h=a-b$ is the distance from the inner sphere to the outer one, and $b$ is the

Correspondence to: V. Roldugin,

E-mail: roldugin@pgi.kolasc.net.ru radius of the outer sphere. Expression (1) can be rewritten as follows (taking into consideration $h \ll a$ )

$f_{n} \equiv \frac{\omega_{n}}{2 \pi} \approx \frac{c}{2 \pi a} \sqrt{n(n+1)}=10.6 \sqrt{\frac{n(n+1)}{2}} \mathrm{~Hz}$,

The simplest model predicts the first order frequency $f 1=10.6 \mathrm{~Hz}$. Observations yield on average $f_{1}=7.8 \mathrm{~Hz}$ (Balser and Wagner, 1962). The frequency became smaller because the ionosphere is not a perfect conductor. Introducing the impedance $Z$ of the upper edge $(r=b)$, the following equation results for the resonance frequencies (providing $|Z| \ll 1, \quad h \ll a$ ) (Bliokh et al., 1980)

$\omega_{n}=\frac{c}{a}\left(\sqrt{n(n+1)-\frac{Z^{2}}{4} \frac{a^{2}}{h^{2}}}+\frac{i Z}{2} \frac{a}{h}\right)$.

If the dielectric permeability at $r>b$ is equal to $\varepsilon=$ const, the impedance is:

$Z=\frac{1}{\sqrt{\varepsilon}}$.

For an isotropic ionosphere, the dielectric permeability has the following form

$\varepsilon=1-\frac{\omega_{0}^{2}}{\omega(\omega-i v)}$

where $\omega$ is the wave frequency, $\omega=\left(4 \pi e^{2} N / m\right)^{1 / 2}$ is the electron plasma frequency, $e$ and $m$ are the electron charge and mass respectively, $N$ is the electron number density, $v$ is the frequency of collision of electrons with neutrals and ions. One can choose such values of $\varepsilon$ and $h$ that several resonance harmonics calculated with the help of Eq. (3) coincide sufficiently well with the observed ones. A more complicated distribution of $\varepsilon$ with the height allows us to explain the observed width of the resonance band related to the resonator quality. The ionospheric $D$ region (heights from 60 to $90 \mathrm{~km}$ ) appears to determine the resonance spectrum.

In the real ionosphere, $\varepsilon$ varies both with the height and in horizontal directions. In addition, the dielectric 
permeability of the ionospheric plasma in the geomagnetic field at heights above $\sim 75 \mathrm{~km}$ is a tensor. Hence $Z$ in Eq. (3) presents some integral parameters.

The waves in the Earth-ionosphere cavity are generated by lightning discharges. At the same time, the Schumann resonance intensity is controlled not only by thunderstorms but also by the solar and geomagnetic activity. Sentman and Fraser (1991) showed the dependence of the observed resonance power on the effective height of the ionospheric $D$ region at the observation point. The dependence of the first and second order resonance modes on solar activity was discovered by Fullekrug and Fraser-Smith (1996). They found 27-day amplitude variations coinciding in phase with the solar spot number. From the measurements made in Hungary, Marcz et al. (1997) found similarities between the resonance amplitude and the atmospheric electric field despite the local character of the latter. The similarities are observed at different temporal scales, from six months to several hours.

Diurnal variations of the resonance frequencies caused presumably by the eccentricity of the geomagnetic dipole were found by Balser and Wagner (1962) at the very start of the investigations. Sao et al. (1973) reveal that the first mode frequency depends not only on the zenith angle of the Sun along the signal propagation path from a lightning flash to the observation point, but also on the planetary magnetic disturbance. Choosing $A p$ as a disturbance index, Sao et al. (1973) found that the second order mode frequency decrease at $0.2 \mathrm{~Hz}$ by $A p$ increases from 0 to 50 . They found also a noticeable positive correlation of the first order mode frequency with 1-8 $\AA$ solar X-ray intensity.

According to Eqs. (3) and (4), the Schumann resonance frequency depends on the dielectric permeability of the ionospheric $\mathrm{D}$ region. The powerful agent disturbing this region is solar protons penetrating into the atmosphere during solar proton events (SPEs). In this study we examine variations in the first order frequency of the Schumann resonance during such events.

\section{Data}

The first resonance frequency behavior has been studied for SPE of November 6, 1997. The event was preceded by another SPE on November 4 related to the class 2 optical flare at a region with heliocoordinates S14 W33. GOES spacecrafts registered an increase of X-ray flux at 0552 UT that maximized at $0556 \mathrm{UT}$ and ended after $7 \mathrm{~h}$. Solar protons with energy more than $10 \mathrm{MeV}$ started at $0830 \mathrm{UT}$, reached a maximum at $1120 \mathrm{UT}$ and ended at 1123 UT on November 5.

On November 6 the flare under consideration 2B/ X9.4 occurred in the region S18 W63 on the Sun. The intensities of the solar X-rays and of the energetic proton flux obtained from GOES- 8 satellite are shown in Fig. 2a. The 1-8 $\AA$ X-ray burst with impulsive profile started at 1149 UT and reached its maximum of $7.5 \cdot 10^{-4} \mathrm{~W} / \mathrm{m}^{2}$ at $1155 \mathrm{UT}$. The proton flux with

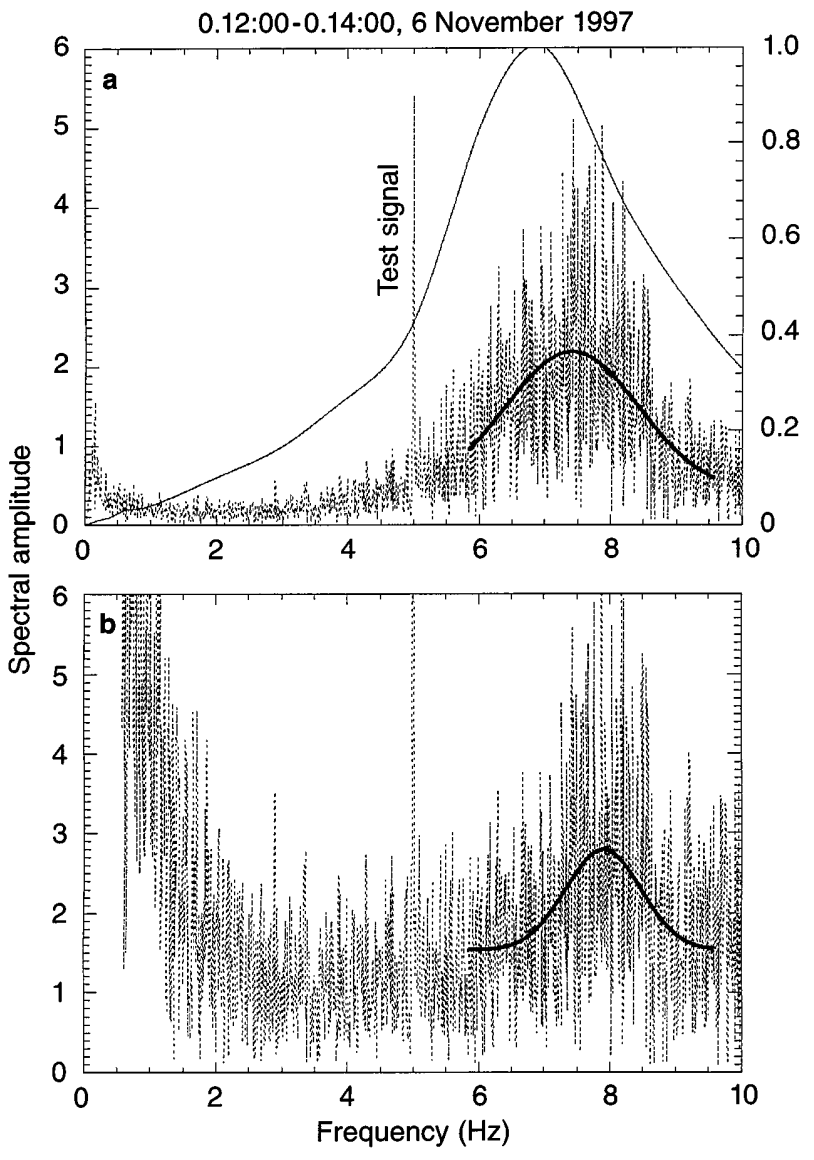

Fig. 1. a The spectrum of 2-min fluxmeter record (dotted line), its approximation by Gaussian function (thick line) and the amplitude versus frequency characteristic of fluxmeter (thin line). b The spectrum after frequency correction and its new approximation

energy of more than $100 \mathrm{MeV}$ started to increase at 1245 UT, maximized at $1640 \mathrm{UT}$ and remained high till 24 h later.

The first Schumann resonance behavior was studied with the help of data from four fluxmeter channels in Lovozero $\left(68.0^{\circ} \mathrm{N}, 35.1^{\circ} \mathrm{E}\right)$. The magnetic induction coils consist of 250000 turns wound over ferrite rods. All the channels have a bandwidth between 1-10 Hz. Two channels with numbers 10 and 11 registered $D$ - and $H$-components correspondingly and two others, 14 and 15 , registered the circularly polarized components, left and right correspondingly. The calibration monochromatic signal advanced at a frequency of $5 \mathrm{~Hz}$. Signals of all four channels were registered in digital form with a 50 ms time step.

To obtain the time variations of the first Schumann resonance frequency, the spectra of two-minute intervals, containing 2400 points, were calculated with the FFT method for all four channels. The typical example is shown in Fig. 1a as a sweeping dotted curve. The thick line is its approximation by a Gaussian function inside the interval of $5.8-9.6 \mathrm{~Hz}$ with four unknown parameters: the frequency of maximum, halfwidth, amplitude and the constant level. The frequency of the maximum is about $7.4 \mathrm{~Hz}$. This too low value and asymmetrical slope structure of the peak are caused by 


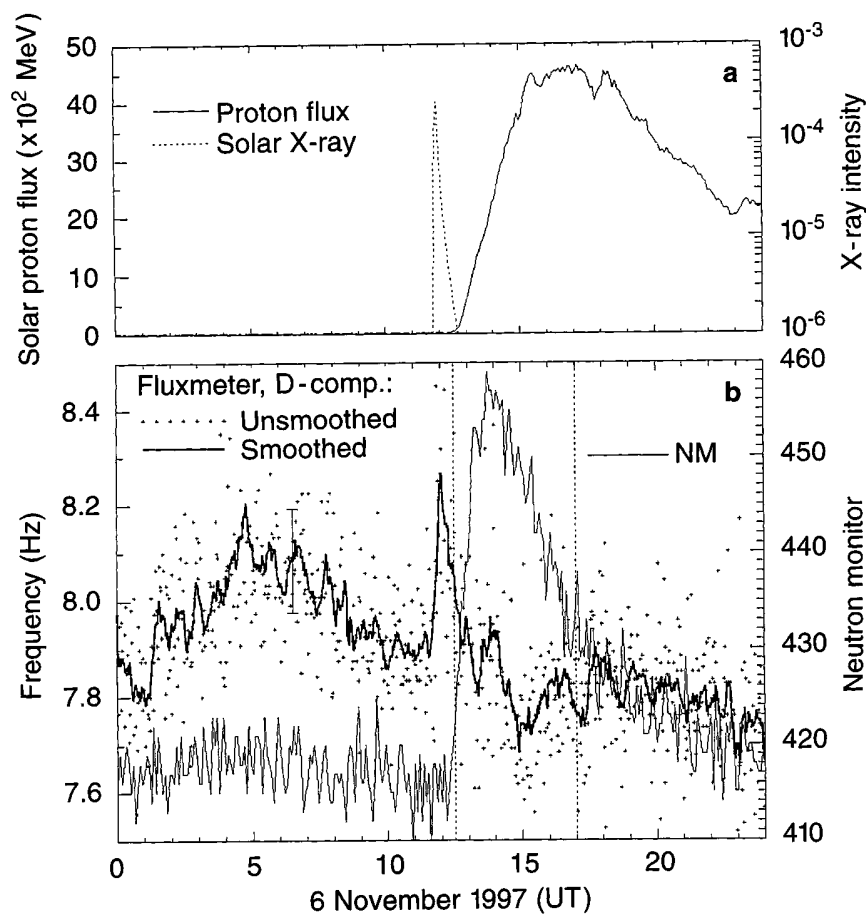

Fig. 2. a Proton $\mathrm{E}>100 \mathrm{Mev}$ flux and solar X-ray $1-8 \AA$ intensity from GOES-8 data. $\mathbf{b}$ The count rate of Apatitian monitor (thin line) on 6 November, 1997 and the variation of frequency of the first Schumann resonance (thick line and points) are shown

the roll-off of low pass filter. The thin line in Fig. 1 is the amplitude versus frequency characteristic of the channel. Its peak is located at $6.9 \mathrm{~Hz}$. To correct the signal, spectra were divided this characteristic, and the Gaussian approximation was applied to improved spectra, see the Fig. 1b).

\section{Experimental results}

Figure $2 b$ shows count rates of the neutron monitor in Apatity, and the first SR frequency in the tenth channel, $D$-component, displayed both by points for every $2 \mathrm{~min}$ interval, and by a running-average smoothed curve. The length of the smoothing is 11 points. The peak of the neutron monitor increase is marked by the dotted vertical lines. Its greatest height exceeds the pre-event background by $10 \%$.

The daily mean value of the frequency in Fig. 2 is about $7.9 \mathrm{~Hz}$. Except for stochastic scattering, there are long-term variations of the frequency. Augmentation of the neutron monitor count rate is accompanied by the resonance frequency decrease. The greatest decrease is between $0.05-0.1 \mathrm{~Hz}$, i.e., about $1 \%$ of the resonance frequency. It can also be seen that the X-ray flare at 1145 UT was accompanied by an increase of the resonance frequency by $0.3 \mathrm{~Hz}$ or by $3.5 \%$.

A statistical scatter of the frequency values is comparable with magnitude of these mentioned effects. One of the causes of the great scatter is a too rough digitizing frequency $\Delta t=0.05 \mathrm{~s}$, so the Nyquist frequency $f_{N}=1 / 2 \Delta t=10 \mathrm{~Hz}$ is only a little greater than the resonance frequency $7.8 \mathrm{~Hz}$. Reliability of the noted frequency deviations may be confirmed by comparison of the frequency variations shown in Fig. 2 with others.

Figure 3 shows all four channels of the fluxmeters. The frequency decrease after 1230 UT can be seen on all of them. The short-lived frequency increase, simultaneous with the solar X-ray burst near $1150 \mathrm{UT}$, is present also on all channels.

Reliability of the first SR frequency decrease after onset of the energetic proton precipitation is confirmed by comparison of the frequency variations in the tenth channel for November 5, 6, and 7 as shown in Fig. 4. For every day the frequency value of $7.8 \mathrm{~Hz}$ is shown by the dashed lines. On all three adjacent days the frequency increase takes place almost at $5 \mathrm{~h}$ UT and after midday the frequency increase to $8.0 \mathrm{~Hz}$ is observed only on November 5 and 7. On the SPE day it is absent, the frequency remaining near $7.8 \mathrm{~Hz}$.

No response of the SR amplitude to the SPE is found. Figure 5 shows the amplitude variations for 5, 6, and 7 November in arbitrary units, determined from Gaussian approximation. The vertical dashed lines pick out the active interval 1230-1700 UT, as in Fig. 2b. The diurnal variations of the SR amplitude for all three days are similar and rather strong (with the magnitude nearly $30 \%$ ). However, the active interval for 6 November shows no essential distinctions from the same intervals for adjacent days.

We have estimated the stochastic scatter of the frequency values in Fig. 2 as the square mean deviation of data in the interval 0500-1100 UT after excluding the linear trend. It is $\sigma=0.11 \mathrm{~Hz}$ and plotted as a bar at 0600 UT. Let us use this value to estimate the significance of the maximum at 1145 . The mean value

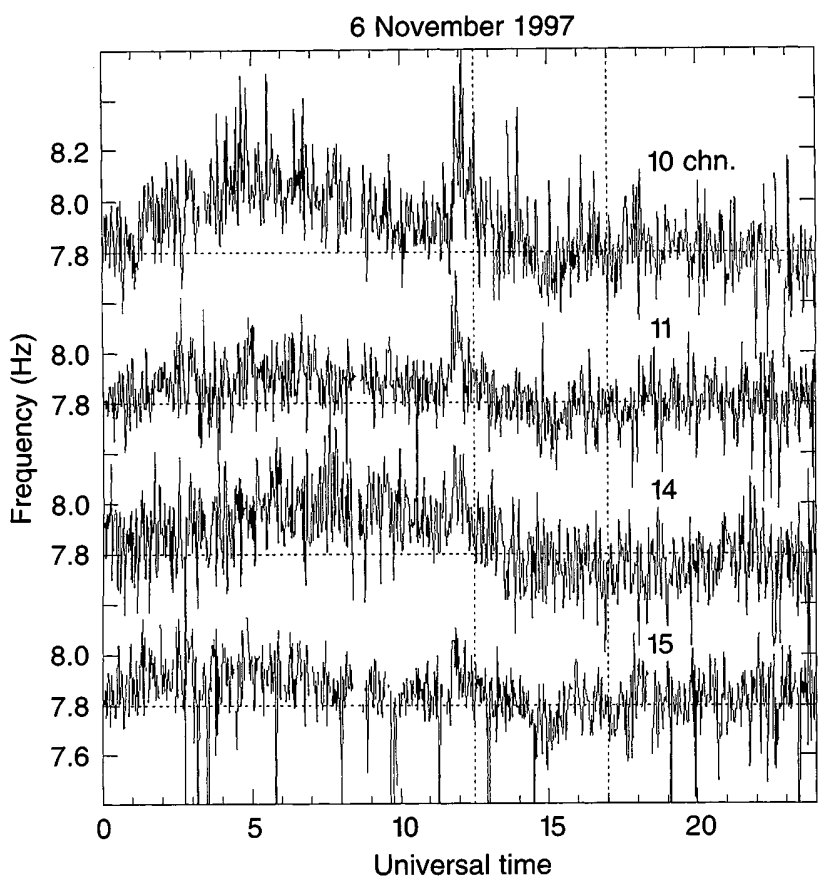

Fig. 3. Variations of the first Schumann resonance frequency on 6 November, 1997 at all four fluxmeter channels 


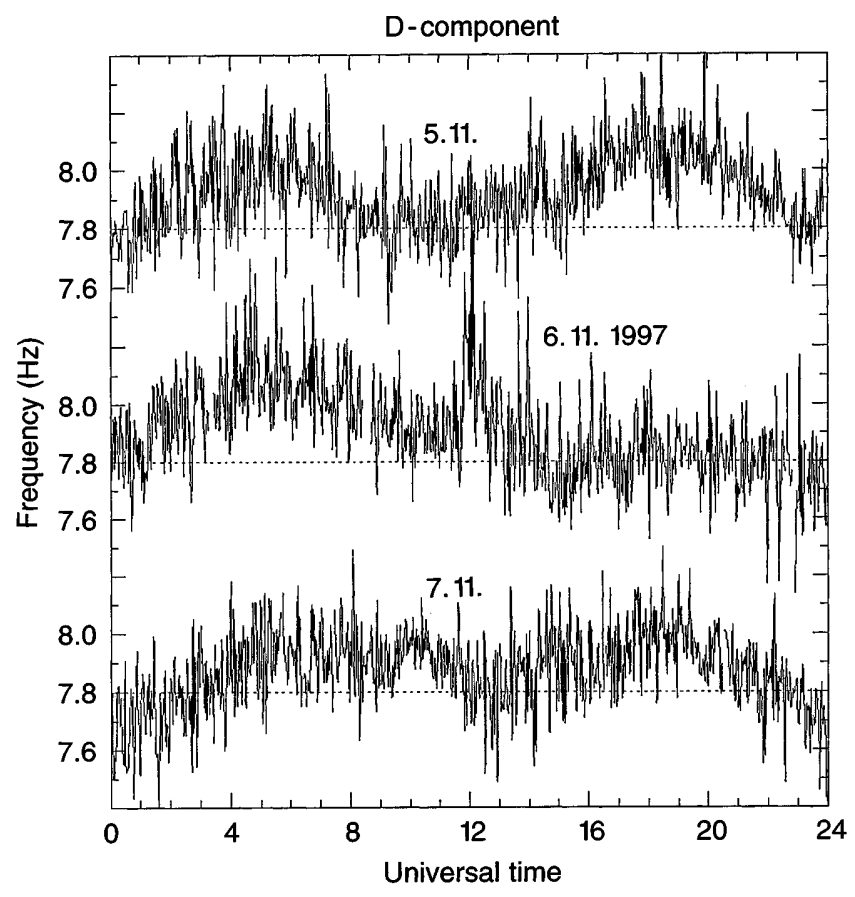

Fig. 4. Variations of the first Schumann resonance frequency on 5, 6, and 7 November, 1997 in the tenth channel

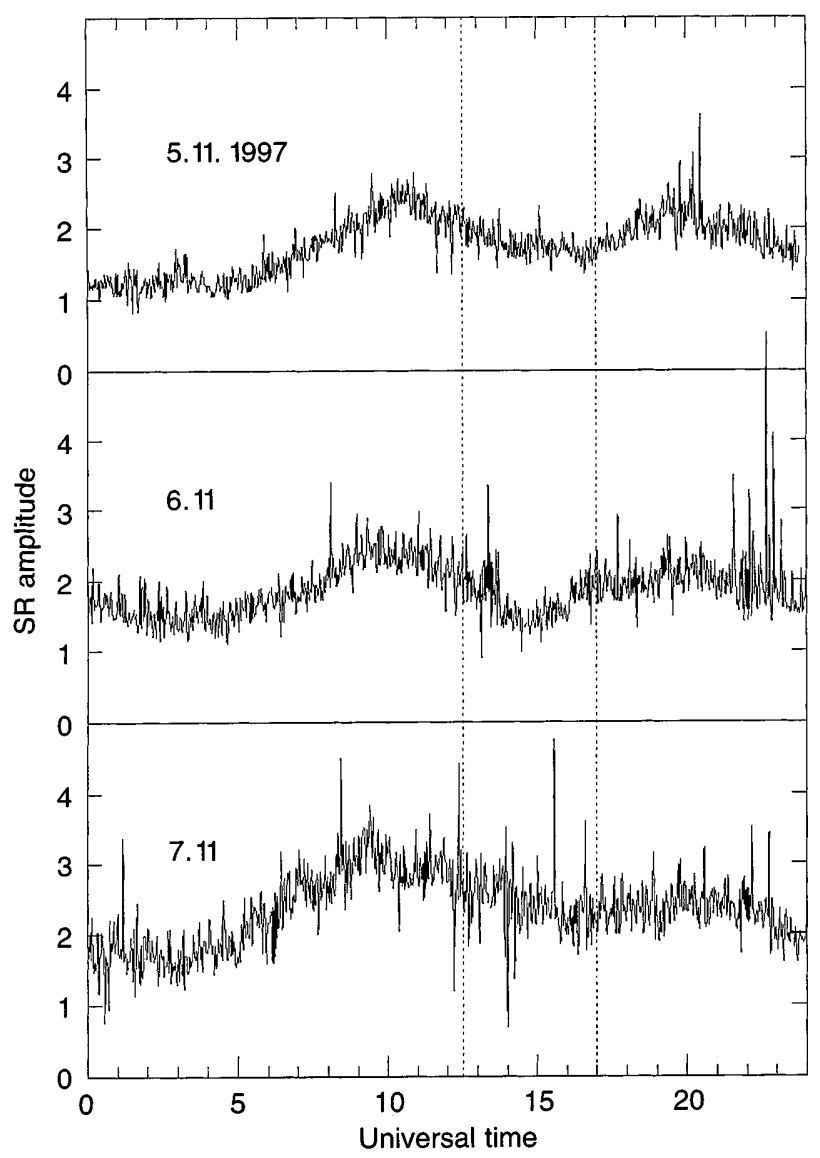

Fig. 5. Variations of the first Schumann resonance amplitude on 5, 6, and 7 November, 1997 in the tenth channel of the frequency for the X-ray burst interval 1146-1214 UT is $8.18 \mathrm{~Hz}$, the number of points in this interval is $n=15$. Thus the difference between the daily mean value of $7.90 \mathrm{~Hz}$ and the mean value of the peak $8.18 \mathrm{~Hz}$ exceeds the standard error $\mathrm{E}=\sigma / \sqrt{n}=0.028$ by exactly 10 times.

\section{Discussion}

Expression (3) allows us to obtain the resonance frequency variation. Assuming $|\varepsilon| \gg 1, v=$ const, $\omega \ll v$, neglecting the second term under the radical, and taking into consideration Eqs. (4) and (5), we obtain the variation of the real part of the frequency:

$\frac{\operatorname{Re}\left(\delta f_{n}\right)}{\operatorname{Re} f_{n}}=\frac{c}{2 \pi a \operatorname{Re} f_{n}}\left(\frac{\delta N}{2 N}+\frac{\delta h}{h}\right) \operatorname{Im}\left(\frac{Z}{2} \frac{a}{h}\right)$.

According to Eq. (3), the first order frequency $\mathrm{Re}$ $f_{1}=7.8 \mathrm{~Hz}$ takes place under the following condition

$\operatorname{Im}\left(\frac{Z}{2} \frac{a}{h}\right) \approx 0.37$.

Substitution of Eq. (7) into Eq. (6) yields the variation of the first order frequency

$\frac{\operatorname{Re}\left(\delta f_{1}\right)}{\operatorname{Re} f_{1}} \approx 0.36\left(\frac{\delta N}{2 N}+\frac{\delta h}{h}\right)$.

Expression (8) shows that the frequency can change due to both electron density variations in the ionospheric D region and variation of the altitude of the D region's lower edge. X-rays and solar protons affect the ionosphere in different ways. Growth of the X-ray flux enhances the electron density without a significant change of the ionosphere altitude. The $3.5 \%$ increase in the Schumann resonance frequency observed at 1155 UT on 6 November 1977 could be caused by the density variation $\delta N / N \approx 19 \%$ averaged over all the Earth. Since X-rays affect the dayside ionosphere only, the observed $3.5 \%$ frequency increase requires $\sim 38 \%$ increase in the dayside electron density. This estimate is within the range of possible density variations during X-ray events. According to calculations by Curto et al. (1994), a solar X-ray burst of class X9.4 raises the electron density at the altitude of $100 \mathrm{~km}$, i.e., near the top edge of the cavity, twice or three times.

Solar protons penetrating deep into the atmosphere ionize regions lower the normal $\mathrm{D}$ region that corresponds to a decrease of the ionosphere altitude. According to Eq. (8), the 1\% decrease in the resonance frequency observed from 1220 to 2000 UT could be caused by the global decrease of the ionospheric altitude $\delta h / h \approx-2.8 \%$. Assuming the protons precipitate at latitudes above $60^{\circ}$, means that the precipitation area is about $1 / 8$ of the whole ionospheric surface and the required altitude variation in the high latitudes is $\delta h / h \approx-2.8 \% \times 8 \approx-22 \%$. If the normal altitude of the Schumann cavity is $h=75 \mathrm{~km}$ we obtain $h \approx 58 \mathrm{~km}$ in high latitudes during the SPE on 6 November, 1977. These estimates are very rough, of course, but 
nevertheless they are consistent with commonly observed ionospheric effects of solar protons (e.g., Mitra, 1974).

\section{Conclusion}

The decrease of the frequency of the first Schumann resonance is found for the relativistic proton precipitation in the SPE of 6 November, 1997. Its value is about $1 \%$. The frequency increase of about $3.5 \%$ coincides with the strong solar X-ray burst lasting $10 \mathrm{~min}$. Both effects are explained by changes of the dielectric permeability in the Schumann cavity.

Topical Editor D. Alcaydé thanks a referee for his help in evaluating these pages.

\section{References}

Balser, M., and C. A. Wagner, On frequency variations of the Earth-ionosphere cavity modes, J. Geophys. Res., 67(10), 40814083, 1962.
Bliokh, P. V., A. P. Nikolaenko, and Ye. F. Filippov, Schumann Resonances in the Earth-Ionosphere Cavity, Peter Perigrinus, London, 1980.

Curto, J. J., C. Amory-Mazaudier, J. M. Torta, and M. Menvielle, Solar flare effects at Ebre: unidimensional physical, integrated model, J. Geophys. Res., 99 (A12), 23 289-23 296, 1994.

Fullekrug, M., and A. C. Fraser-Smith, Further evidence for a global correlation of the Earth-ionosphere cavity resonances, Geophys. Res. Lett., 23 (20), 2773-2776, 1996.

Marcz, F., G. Satori, and B. Zieger, Variations in Schumann resonances and their relation to atmospheric electric parameters at Nagycenk station, Annales Geophysicae, 15 (12), 1604-1614, 1997.

Mitra, A. P., Ionospheric effects of solar flares. Astrophysics and space science library, 46. D. Reidel, Dordrecht 266 p., 1974.

Sao, K., M. Yamashita, S. Tanahashi, H. Jindon, and K. Ohta, Experimental investigations of Schumann resonance frequencies, J. Atmos. Terr. Phys., 35 (11), 2047-2053, 1973.

Schumann, W. O., Über die Stralungslosen Eigenschwingungen einer leitenden Kugel die von Luftscicht und einer Ionosphärenhûlle umgeben ist, Z. Naturforsch., 7a, 149-154, 1952.

Sentman, D. D., and B. J. Fraser, Simultaneous observations of Schumann resonances in California and Australia: evidence for intensity modulation by local height of the D-region, J. Geophys. Res., 96 (A9), 15 973-15 984, 1991. 